\title{
Research on the Window Display Design of the Modern Brand Clothing
}

\author{
Zhou Liping \\ Jiujiang university, Jiujiang 332005, China \\ Xuexi123@163.com
}

Keywords: Personalized fitting; Brand clothing; Window display; Digital; Diversification.

\begin{abstract}
Based on the clothing industry diversification, virtualization, digital development characteristics and clothing electronic commerce development needs, this paper proposed a personalized fitting system based on 3D human body, the window display system can release the latest clothes, users can input the their own feet inch parameters and choose different size models of the human body. The model can try on new clothes and view the clothes effect, thus strengthening the advertising display 3D effect of clothing brand. This technology can be very convenient to meet the garment customization consumer demand of fitting, and can easily modify garment according to the requirements of the consumers, it lets consumers participate in the clothing design and adapt to the requirements of economic experience.
\end{abstract}

\section{Introduction}

After burrowing life, Human's economic life goes through the agricultural economy, industrial economy and service economy, and at last comes to the experience economy. The so-called experience economy is personalized consumption and generation, some economists regarded it as the fourth industry, and its characteristics are the large-scale tailored [1-3]. With economic and social development and high level of people's living, spiritual and cultural life of the demand also continues to improve [4]. Properties of clothing have a close relationship with human self cognition and cultural quality. In the clothing display system, people like to get rid of the stale and bring forth the fresh, reject the parrot and like personality. Therefore, the design of new personality window display advertising system has important significance for sales and art of clothing display.

\section{Development Trend of Modern Brand Clothing}

Clothing diversification. According to the ZitexStore online survey data, $77.9 \%$ consumers demand the garment fit, 39.1\% require the quality of clothing, 37.1\% demand clothing personality $[5,6]$. From this it can be seen, consumers pursuits more and more the diversity and individuality, the mass production mode of clothing has no longer meet the needs of the market. While the display advertising system should be more personalized and diversified elements to meet the needs of consumers.

Digital clothing. With the development of computer science and technology, high volume and tailoring, and personalized three-dimensional fitting using virtual technology can greatly reduce the cost of tailor, shorten the production cycle [7]. Digital fashion can use modern brand clothing display window, so that consumers can specify the virtual fitting model and put on the virtual digital garment according to their different body size parameters, which shows customers new clothing brand, and improves the clothing art and the stereo effect.

Electronic commerce. Network store has a lot of advantages comparing with the traditional store, such as rich network products, and the price is relatively cheap, easy to buy, express delivery can be delivered at any time, so that people's way of life has changed greatly. Because the network is convenient and fast service, so that the network shopping is becoming the hot spot, so the new window system should adapt to this trend. 


\section{Modern Brand Clothing Window Advertisement Display System}

In order to make the brand clothing window display system having a personalized and stereo effect, based on the basic functions of the V-Stitcher, this paper designs personalized clothing virtual fitting and display system, and V-Stitcher is applied to the effect of tailor, which improves the clothing of digital set and the 3D virtual display function [8-10]. The system overall design framework is as shown in Figure 1.

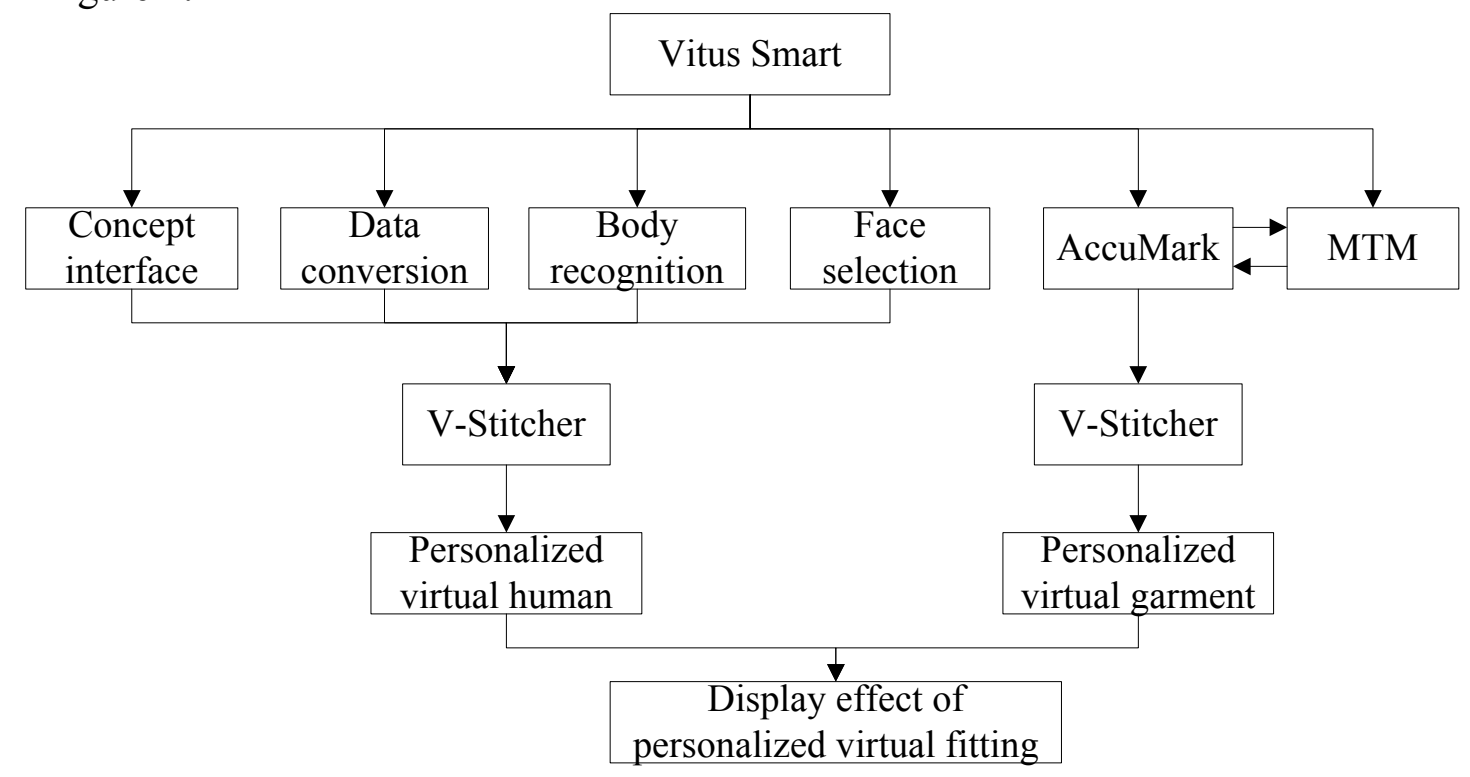

Fig. 2 The design scheme of brand clothing window display

Figure 1 shows the design scheme of brand clothing window display. In the current computer conditions, it can be fast and realistic to generate a similar virtual human body model. In order to strengthen the approximation effect of the human body's feature, it divides the female body, using the clustering algorithm to partition the physical features. Assuming that the sample set of clustering is:

$X=\left(x_{1}, x_{2}, \cdots x_{n}\right)$.

Assume that each individual sample has $m$ index, the characteristic vector of the sample index $j$ is:

$x_{j}=\left(x_{1 j}, x_{2 j}, \cdots x_{m j}\right)^{T}$.

The $n$ feature vector of sample index can be expressed as in matrix:

$X=\left(X_{i j}\right)_{m x n}$.

Among then, $x_{i j}$ is $i$ feature vector of $j$ sample's indicator, $i=1,2,3, \cdots, m ; j=1,2,3, \cdots, n$. The membership functions and the optimization of the membership matrix is:

$$
\begin{aligned}
& r=r(x) . \\
& R=\left(r_{i j}\right)_{m x n} . \\
& r_{i j}=r\left(x_{i j}\right) .
\end{aligned}
$$

By using $m$ index, it can get the fuzzy recognition matrix by using $m$ classification.

$$
U=\left(u_{h j}\right)_{c \times n} .
$$

Among then, $u_{h j}$ is the $h$ relative membership degree of the $j$ sample, $h=1,2,3, \cdots, c$, and it meets the meet the following conditions: 


$$
\begin{gathered}
0 \leq u_{h j} \leq 1 . \\
\sum_{h=1}^{c} u_{h j}=1 . \\
\sum_{j=1}^{n} u_{h j}>0 .
\end{gathered}
$$

Assuming that $m$ index cluster centers are characteristic values in the category of $h$, then the $c$ index characteristic value can be expressed as a fuzzy clustering center matrix.

$$
S=\left(s_{i h}\right)_{m x c} \text {. }
$$

Among then, $s_{i h}$ is $i$ normalized values of characteristic value with $h$ class, and it meets the requirements $0 \leq\left(s_{i h}\right) \leq 1$.

In order to realize the modification of the human body characteristic parameters, it uses VB programming style to copy, delete and rename operations the window fitting model incarnation, so as to achieve the application programming interface [11-13]. Through the input parameter, it changes the incarnation of the model function, and the main program is as follows:

Copy Avatar:

Private Sub Command1_Click()

FileCopy “E:|Program Files\VStitcher\Avatar\AvatarDeflnew_kam_f.adf” ,

"E: $\backslash 1 \backslash$ Avatar $\backslash$ AvatarDeflnew_kim_f.adf"

\section{End Sub}

Delete Avatar:

Private Sub Command6_Click()

KILL "E: Program Files\VStitcher\Avatar\AvatarDeflnew_kam_f.adf"

End Sub

Modify avatar parameters:

Private Sub Command6_Click()

Using the programming software, it can modify the various parameters of the human body to achieve the fitting effect of the human body [14]. The user can select fitting model according to their size parameters, which can be input parameters including height, chest circumference, waist circumference, hip circumference and arm length.

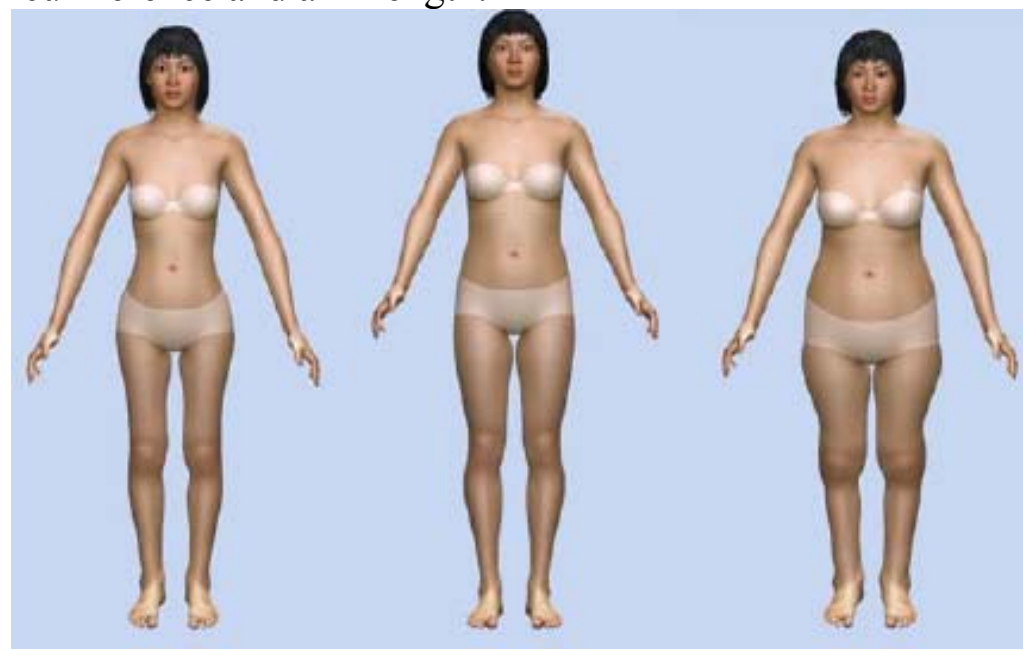

Fig.2 Display fitting model with different input parameters 
Figure 2 shows by inputting different characteristics of human body size, through the virtual simulation; window display body fitting model is get. The users can choose different mannequins fitting according to their different feature sizes.

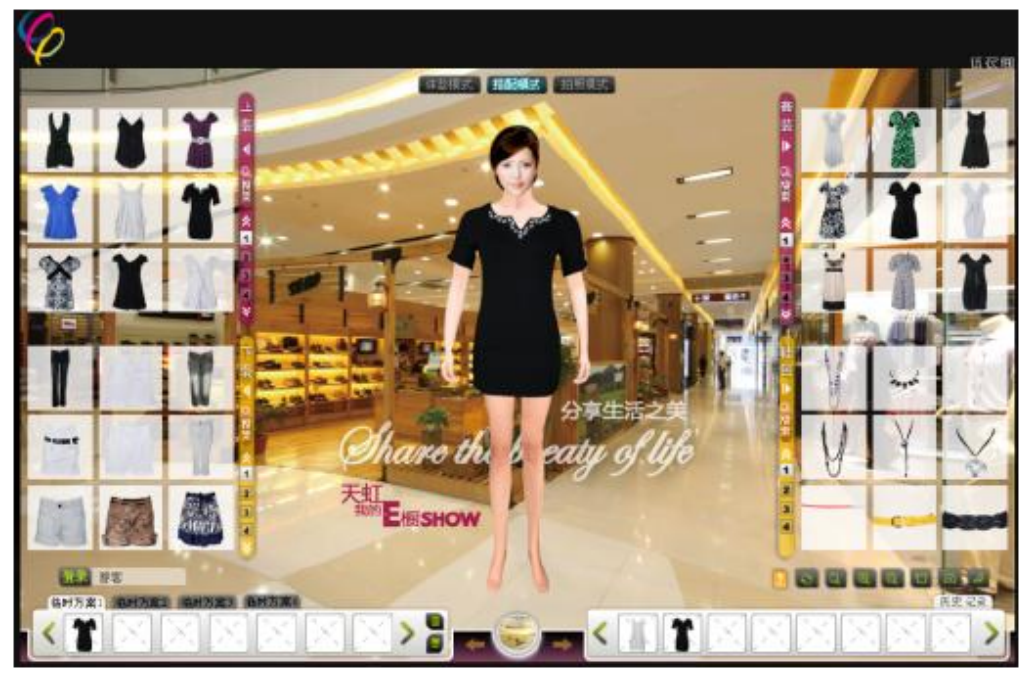

Fig. 3 Modern brand clothing window display system

Figure 3 shows the designed modern network window display system, it can release the latest clothes, and model can try it on to see the effect of clothes, so as to strengthen the 3D effect of brand clothing ads show.

\section{Test Results Show based on Fitting System Window Advertising}

In order to verify the effect of the designed modern brand clothing display system, this paper statistics online store sales and the reason of customer into the shop, and compared results before and after using window display system [15]. The reason of the customer coming into the store is as shown in Figure 4.

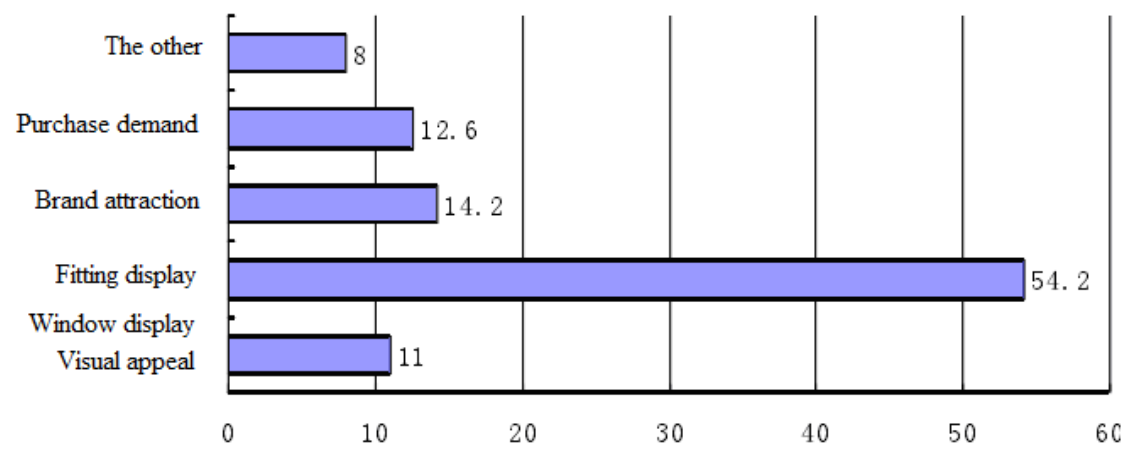

Fig. 4 Statistics on the reasons for the customer into the store after using the new window

Figure 4 shows statistics on the reasons for the customer into the store after using the new window. From the picture it can be seen, the $54.2 \%$ of customers into the shop to buy clothes is attracted by the fitting showcase, the brand identity in the proportion of stores is $14.2 \%$, demand to buy into the store is $12.6 \%$. Illustrated by these data, most of the customers into the store is meaningless, and the fitting window display advertising system trigger the customer into the store and buy clothing, which replicated the good results of the new window. 


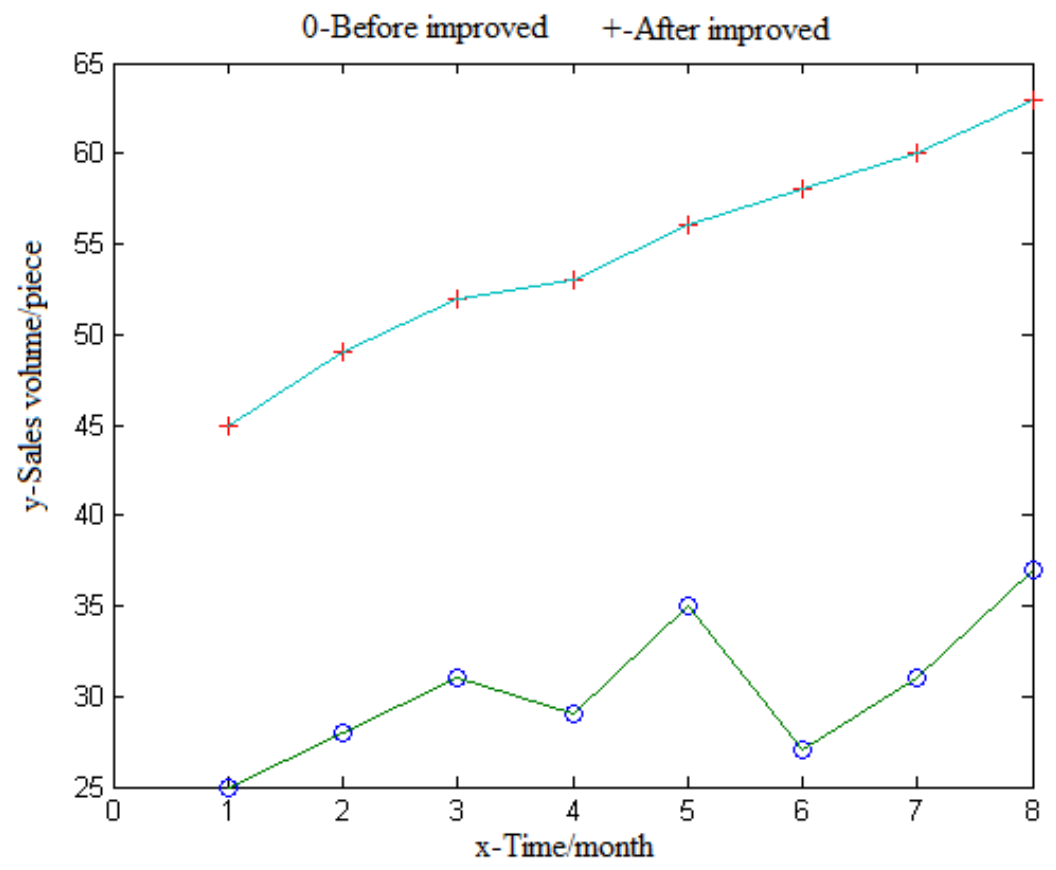

Fig. 5 Apparel sales statistics

In order to further verify the effectiveness of the new window display system, this paper statistics the sales before and after 8 months using the new window. From the results it can be seen, each month's sales ratio has been significantly increased, and the sales volume has different range of growth per month, thus validating the new window system to promote sales.

\section{Summary}

According to the fuzzy clustering algorithm, it divides the human body feature sizes, and based on 3D human body personalized fitting software, this paper designs a new digital clothing shop window advertising display system, the system user can input their own body size parameters and choose different size models, by fitting model to see the effect of new clothing. Finally the clothing window display system is tested. By the test results it can be seen, $54.2 \%$ of customers into the shop to buy clothes is attracted by the fitting showcase, and after using the new window advertising, sales of each month have increased significantly, and sales per month over the last month have different rate of growth, which verifies the effectiveness of the modern clothing brand cabinet advertising display system.

\section{References}

[1] X. Ceng, Z. Jin. The advantages and disadvantages of garment CAD application. Journal of Yulin Normal University, 2012, 3(4): 98-102.

[2] O.Y. Zhang, X.J. Xing. The influence of the OPPLE art culture on the design of modern fabrics and clothing. Silk, 2013, 4(9): 72-78.

[3] J. Liu, Y. Jin. 3D clothing fitting based on VC6.0 and OpenGL. Research of computer Application, 2013, 25 (12): 3825-3831.

[4] Y.L. Lu, R.Q. Li, J.L. Hu. Development history and present status of virtual apparel. Textile journal, 2013, 26 (1): 132-134.

[5] X.R. Hu, S.Q. Cui, H. Wang. Oriented virtual apparel fitting digital human modeling technology. Textile herald, 2014, 4(7): 150-151.

[6] L. Gu, X. Zhang. Overview of 3D human body modeling method in garment. Knitting industry, 2014, 3( 5): 36-38. 
[7] Y.Q. Chen, L.H. Peng. The 3D human body and garment modeling technology. Textile Leader, 2013, 4(6): 112-115.

[8] H. Dai, L. Yang. The size of garment standard classification application research. Knitting industry, 2013 3(11): 19-21.

[9] G.L. Chen, G.Z. Sun, Y. Xu. Research on parallel algorithms. Computer, 2013, 31(9): 1493-1502.

[10] L.F. Lv, J.W. Zhang, J.Z. Sun. Overview of network security visualization. Computer applications, 2013, 28 (8): 1924-1925.

[11] Y. He, W.Q. Wang, F. Xue. Research on massive data mining based on cloud computing. Computer technology and development, 2013, 4(2): 69-72.

[12] Y. Ding, Q.P. Yang, Y.M. Qian. Research on data mining platform based on cloud computing platform and its key technology. ZTE technology, 2013,19(1): 53-60.

[13] K. Li, Z. Chang. The design and implementation of parallel data mining system based on cloud computing. Micro computer information, 2013, 27(6): 121-123.

[14] G.L. Sun, H.L. Qi. Spam filtering based on online sorting logic regression. Journal of Tsinghua University, 2013, 53(5): 734-740.

[15] B.T. Liu. Research on data mining algorithm based on rough set. China West technology, 2013, 10(14): 11-12. 